Interdisziplinäre Anlaufstelle

\section{Neue Schwindelambulanz in München eröffnet}

- Am Integrierten Forschungs- und Behandlungszentrums für Schwindel, Gleichgewichts- und Augenbewegungsstörungen IFBLMU in München (Direktor: Prof. Thomas Brandt) wurde am 3. Februar 2011 die neue Schwindelambulanz eröffnet. Sie ist eine weltweit einzigartige interdisziplinäre Anlaufstelle für Patienten mit Schwindelsymptomen. Keine andere Schwindelambulanz vereint so viele Disziplinen (Neurologie, HNO, Ophthalmologie, Psychosomatik, Radiologie, Neuroradiologie, Nuklearmedizin, Innere Medizin, Neuropädiatrie) unter einem Dach und bietet dadurch Hilfe auch für Personen mit schweren oder ungewöhnlichen Schwindelformen. Die Behandlung der Patienten erfolgt hier nach gemeinsamen Leitlinien zu Diagnose und Therapie.

Die neu eröffnete Schwindelambulanz bündelt die bereits bestehenden Ambulanzen personell und räumlich an einem Ort und bildet eine Einheit in der von der Erstdiagnostik über die Therapie bis hin zu Verlaufskontrollen eine umfassende Betreuung von Schwindelpatienten erfolgen kann. Jährlich sollen hier etwa 3.000 Patienten behandelt werden.

Im Anschluss an die Eröffnung der Schwindelambulanz gaben Expertinnen und Experten auf der Fachkonferenz „Forschung konkret: Dem Schwindel auf der Spur" Einblicke in die Arbeit und Forschungsschwerpunkte des IFB LMU für Schwindel, Gleichgewichts- und Augenbewegungsstörungen.

Mit den „Integrierten Forschungs- und Behandlungszentren (IFB)“ fördert das Bundesministerium für Bildung und Forschung (BMBF) die enge Verknüpfung von Forschung und Patientenversorgung. Mit einem IFB soll in einem bedeutsamen Krankheitsbereich der nachhaltige Aufbau eines Forschung und Versorgung umfassenden Zentrums

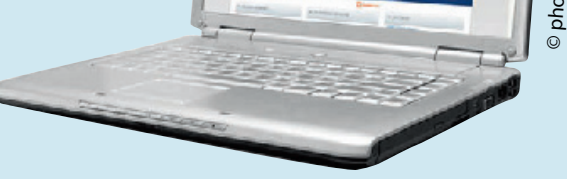

Nachrichten auf www.SpringerMedizin.de

\section{Die Top 3 im März}

\section{Erwartungshaltung entscheidend: Erheblicher} Placeboeffekt bei Opioden

\section{Erschreckende Entwicklung: Lebensmittelallergien} bei Kindern nehmen rasant zu

\section{Folsäuremangel schadet dem Gehör! Mit Kohl und Bananen gegen Altersschwerhörigkeit}

Diese drei Beiträge wurden im vergangenen März im Fachportal HNO von SpringerMedizin.de am häufigsten angeklickt. Zum Nachlesen loggen Sie sich ein und geben Sie die jeweiligen Schlagworte in die Suche ein. Springer. Medizin.de bietet Ihnen täglich neue Informationen aus der ganzen Welt der Medizin.

zu einem gemeinsamen Schwerpunkt gestärkt werden. Durch die Überwindung traditioneller Fachgrenzen kann in einem IFB interdisziplinär auf hohem Niveau gearbeitet werden. Patienten sollen künftig noch schneller von neuen Forschungsergebnissen profitieren. Ziel ist auch die Verbesserung der Nachwuchsförderung, um hochqualifizierte Expertinnen und Experten in Deutschland zu fördern und zu binden und den Forschungsstandort Deutschland zu stärken.

\title{
Riskante Praxis
}

\section{Die Dosis von geteilten Tabletten kann stark variieren}

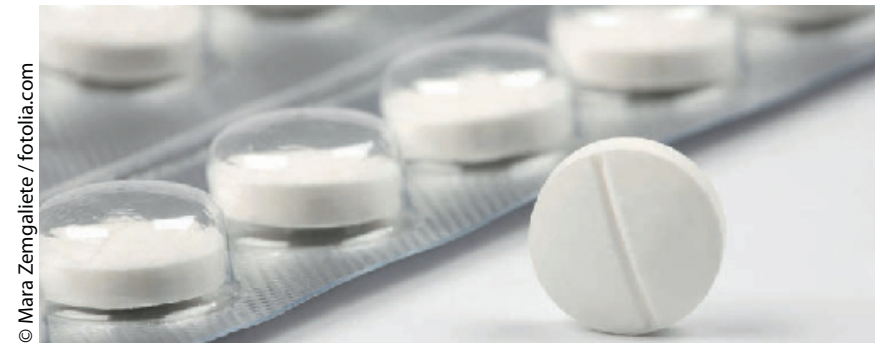

- Tabletten zu teilen, kann riskant sein, vor allem bei Medikamenten mit einem engen Bereich zwischen therapeutischer und toxischer Dosis. Denn je nach Tablettenart und Teilmethode kann es zu Dosisabweichungen kommen, wie belgische Wissenschaftler herausfanden.

Fünf Freiwillige im Alter zwischen 21 und 55 Jahren wurden aufgefordert, acht in der Größe und im Aussehen unterschiedliche Tabletten zu halbieren oder zu vierteln. Nur einer von ihnen hatte Erfahrungen im
Tablettenteilen. Die Tabletten wurden mithilfe eines Tablettenteilers (Gruppe 1), einer Schere, wenn die Tablette keine Kerbe hatte oder von Hand, wenn die Tablette eine Kerbe hatte (Gruppe 2), oder mit einem Küchenmesser (Gruppe 3) geteilt.

Anschließend wurde das Gewicht der Tablettenstücke ermittelt: $31 \%$ wichen mehr als $15 \%$ von ihrem theoretischen Sollgewicht ab und $14 \%$ mehr als $25 \%$. Die genauesten Ergebnisse wurden mit dem Tablettenteiler erzielt. Trotzdem wichen auch hier noch $21 \%$ der Teilergebnisse mehr als $15 \%$ und $8 \%$ mehr als $25 \%$ vom zu erzielenden Soll ab. Einer deutschen Studie zufolge werden mehr als ein Viertel aller Tabletten geteilt, um die Dosisflexibilität zu erhöhen, damit die Tabletten besser zu schlucken sind oder um Kosten zu sparen (für die Patienten, aber auch für die Krankenkassen). Doch die Tablettenteile sind oft ungleich groß und es gehen - je nach Methode - auch mal Stücke verloren. Daran sollte man unbedingt denken. Die Studienautoren fordern zudem die Arzneimittelhersteller auf, in Zukunft ein breiteres Dosisspektrum der einzelnen Medikamente oder flüssige Alternativen anzubieten. ke 